\title{
Categorizing Health Outcomes and Efficacy of mHealth Apps for Persons With Cognitive Impairment: A Systematic Review
}

Daniel R Bateman ${ }^{1,2}$, MD; Bhavana Srinivas ${ }^{1,3}$, MS; Thomas W Emmett ${ }^{4}$, MD, MLS; Titus K Schleyer ${ }^{5,6}$, DMD, PhD; Richard J Holden ${ }^{1,3,6}$, PhD; Hugh C Hendrie ${ }^{1,2}$, MBChB, DSc; Christopher M Callahan ${ }^{1,5}$, MD

\footnotetext{
${ }^{1}$ Indiana University Center for Aging Research, Regenstrief Institute, Inc, Indianapolis, IN, United States

${ }^{2}$ Department of Psychiatry, Indiana University School of Medicine, Indianapolis, IN, United States

${ }^{3}$ School of Informatics and Computing, Indiana University-Purdue University Indianapolis, Indianapolis, IN, United States

${ }^{4}$ Ruth Lilly Medical Library, Indiana University School of Medicine, Indianapolis, IN, United States

${ }^{5}$ Department of Medicine, Indiana University School of Medicine, Indianapolis, IN, United States

${ }^{6}$ Center for Biomedical Informatics, Regenstrief Institute, Inc, Indianapolis, IN, United States
}

Corresponding Author:

Daniel R Bateman, MD

Indiana University Center for Aging Research

Regenstrief Institute, Inc

1101 West 10th Street

Indianapolis, IN, 46202

United States

Phone: 13172749334

Fax: 13172749307

Email:darbate@iupui.edu

\section{Abstract}

Background: Use of mobile health (mHealth) apps is growing at an exponential rate in the United States and around the world. Mild cognitive impairment (MCI), Alzheimer disease, and related dementias are a global health problem. Numerous mHealth interventions exist for this population, yet the effect of these interventions on health has not been systematically described.

Objective: The aim of this study is to catalog the types of health outcomes used to measure effectiveness of mHealth interventions and assess which mHealth interventions have been shown to improve the health of persons with MCI, Alzheimer disease, and dementia.

Methods: We searched 13 databases, including Ovid MEDLINE, PubMed, EMBASE, the full Cochrane Library, CINAHL, PsycINFO, Ei Compendex, IEEE Xplore, Applied Science \& Technology Source, Scopus, Web of Science, ClinicalTrials.gov, and Google Scholar from inception through May 2017 for mHealth studies involving persons with cognitive impairment that were evaluated using at least one quantitative health outcome. Proceedings of the Annual ACM Conferences on Human Factors in Computing Systems, the ACM User Interface Software and Technology Symposium, and the IEEE International Symposium on Wearable Computers were searched in the ACM Digital Library from 2012 to 2016. A hand search of JMIR Publications journals was also completed in July 2017.

Results: After removal of duplicates, our initial search returned 3955 records. Of these articles, 24 met final inclusion criteria as studies involving mHealth interventions that measured at least one quantitative health outcome for persons with MCI, Alzheimer disease, and dementia. Common quantitative health outcomes included cognition, function, mood, and quality of life. We found that $21.2 \%$ (101/476) of the fully reviewed articles were excluded because of a lack of health outcomes. The health outcomes selected were observed to be inconsistent between studies. For those studies with quantitative health outcomes, more than half $(58 \%)$ reported postintervention improvements in outcomes.

Conclusions: Results showed that many mHealth app interventions targeting those with cognitive impairment lack quantitative health outcomes as a part of their evaluation process and that there is a lack of consensus as to which outcomes to use. The majority of mHealth app interventions that incorporated health outcomes into their evaluation noted improvements in the health of persons with MCI, Alzheimer disease, and dementia. However, these studies were of low quality, leading to a grade C level of evidence. Clarification of the benefits of mHealth interventions for people with cognitive impairment requires more randomized controlled trials, larger numbers of participants, and trial designs that minimize bias. 
Trial Registration: PROSPERO Registration: PROSPERO 2016:CRD42016033846; http://www.crd.york.ac.uk/PROSPERO/ display_record.asp?ID=CRD42016033846 (Archived by WebCite at http://www.webcitation.org/6sjjwnv1M)

(J Med Internet Res 2017;19(8):e301) doi: 10.2196/jmir.7814

\section{KEYWORDS}

mHealth; mobile health; applications; Alzheimer disease; dementia; systematic review

\section{Introduction}

Industry analysts expect worldwide mobile phone app users to increase from 2.6 billion in 2015 to 6.1 billion users by 2020 $[1,2]$. Similarly, analysts forecast worldwide mobile device app downloads and usage to grow from 111.2 billion in 2015 to 284.3 billion by 2020 [3]. From a financial scope, global mobile app gross revenue for 2016 surpassed US $\$ 51$ billion and by 2020 is expected to exceed US $\$ 101$ billion [3]. The National Institutes of Health (NIH) Consensus Group on mHealth defines mobile health (mHealth) as "the use of mobile and wireless devices to improve health outcomes, health care services, and health research" [4,5]. The NIH Strategic Plan for 2016-2020 incorporates the study of mHealth technologies and their ability to help prevent and treat illness as a research priority [6]. Following the aforementioned NIH definition of mHealth, a mHealth app operates on either a mobile or wireless device, with an objective of improving health outcomes, health care services, or health research [4].

The mHealth technologies and apps that help persons with mild cognitive impairment (MCI), Alzheimer disease, and dementia offer a unique opportunity for intervention because there are no disease-modifying agents for Alzheimer disease and related dementias [7]. More than 5.4 million people in the United States live with Alzheimer disease, the most common type of dementia [8]. Scientists predict the number of people with Alzheimer disease in the United States to reach 8.4 million by year 2030 [8]. Until disease-modifying agents are found, innovative psychosocial interventions, including mHealth interventions, offer the greatest potential for improving quality of life for persons with dementia and their caregivers [9].

Much remains unknown about the health outcomes used in mHealth apps and the effectiveness of these apps in improving the health of persons with MCI, Alzheimer disease, and dementia. There are literally hundreds of mobile apps that persons with MCI or dementia can use. Those mHealth apps are being marketed to help persons with cognitive impairment with unclear validity to their claims. Persons with cognitive impairment are already using mHealth apps, and will continue to do so in greater numbers, yet often the risks and benefits are not fully understood [3]. Similarly, the effects of these apps on persons with MCI, Alzheimer disease, and dementia have not been adequately reviewed and summarized in a systematic fashion. Therefore, the primary aim of this systematic review seeks to catalog the types of quantitative health outcomes utilized in these mHealth app studies. The secondary aim of this review strives to evaluate the effectiveness of mHealth apps in improving the health outcomes of persons with MCI, Alzheimer disease, and dementia through a review of the current scientific literature.
Background on the types of mHealth interventions included in this review are listed subsequently. These interventions can be grouped into a number of different categories, including cognitive training and serious games, wandering and wayfinding, reminiscence therapy, prompts and multicomponent interventions, engagement interventions, and exercise interventions.

\section{Types of Interventions}

\section{Cognitive Training and Serious Games}

There exists a great deal of interest in using computerized cognitive training as an intervention to prevent and treat neurodegenerative disorders. Rebok et al [10] showed that independent older adults who underwent computerized cognitive training retained cognitive and functional benefits 10 years out from the intervention. However, the potential benefits for persons with MCI, Alzheimer disease, or persons with dementia is much less clear. A recent systematic review found that persons with MCI who received cognitive training had improvements in cognition, whereas persons with dementia had limited evidence for efficacy [11].

Serious games are games with a primary purpose other than entertainment, enjoyment, and fun [12]. They often include cognitive training or exercise training in the form of games. An example of this could be seen with a patient recovering from a stroke playing a serious game involving the activity of swinging a baseball bat in a virtual game rather than doing traditional exercises.

\section{Wandering and Wayfinding}

Wandering is a very common problem in older adults with mild-to-moderate stages of dementia. Such behavior usually occurs as a result of memory deficits and spatial disorientation, which makes persons with dementia less likely to recognize the route [13]. Navigation systems, such as satellite navigation (Global Positioning System), three-dimensional maps, and electronic maps, could provide assistance in locating the patient irrespective of the closed or outdoor environment, and could also support the person with dementia in finding their way back home $[13,14]$. This practice of finding one's way back home or to a preselected destination is known as "wayfinding."

\section{Reminiscence Therapy}

Reminiscence therapy works under the assumption that remote memory remains intact until later in the course of dementia and that recalling and discussing past events and life experiences can help the psychological wellness and cognition of people with dementia [15]. Often reminiscence therapy therapists will utilize music, pictures, art, and other aids in sessions. A therapist or a staff member trained in reminiscence therapy leads the session, which can take either a group or an individual therapy 
format. Reminiscence therapy has been shown to improve well-being, patient-caregiver relations, global cognition, and decrease social withdrawal [16-19].

\section{Prompts and Multicomponent Interventions}

With the progression of disease, persons with dementia lose their ability to perform activities of daily living (ADL) and often require frequent support and assistance from a family member or caregiver [20]. Prompts incorporate a unique approach to support and provide assistance to persons with cognitive impairment. Studies have shown that prompts can help persons with dementia to be less dependent on caregivers [21]. The function of prompts can range from reminders to take medications, to notifications of a scheduled activity, to a verbal or visual cue to get dressed or shower. We combined the prompt category and multicomponent intervention category because there was significant overlap between these two. Multicomponent interventions typically included prompts and some notification system for the caregiver. Other examples of components involve patient location, a communication system with health care professionals, and engagement activities.

\section{Engagement Interventions}

Past studies demonstrated that recreational activities and engagement can lead to persons with dementia having more positive affect, decreased agitation, and decreased passivity [22,23].

\section{Exercise Intervention}

Exercise training has been shown to reduce behavioral and psychological symptoms of dementia [24], to slow progression of cognitive decline in MCI [25], and to lead to increased hippocampus size [26], a region of the brain responsible for short-term memory. A recent review examining the evidence for physical and cognitive interventions to improve brain health found sufficient evidence that both physical and cognitive interventions lead to enhanced neuroplasticity and prevention of pathological aging (MCI, Alzheimer disease, and dementia) [27]. Evidence also suggests that the combination of physical and cognitive interventions may amplify these positive effects on neuroplasticity [27].

\section{Methods}

Using the Preferred Reporting Items for Systematic Review and Meta-Analyses (PRISMA) checklist, we systematically reviewed the scientific literature to find mHealth apps that sought to improve health outcomes of persons with MCI, Alzheimer disease, and dementia. The PRISMA guidelines provide a standardized structure for the design, iterative process, extraction, and synthesis that take place during the development of a systematic review [28]. Aim 1 of this systematic review attempts to catalog quantitative health outcomes used to evaluate mHealth apps, whereas aim 2 seeks to assess the effectiveness of mHealth apps for persons with cognitive impairment that incorporate at least one quantitative health outcome. The systematic review protocol was registered with PROSPERO, the international prospective register of systematic reviews, at inception to avoid duplication [29].

\section{Data Sources and Searches}

A comprehensive search of the literature was performed by a medical librarian (TWE) in Ovid, MEDLINE, PubMed, EMBASE, the full Cochrane Library, Cumulative Index to Nursing and Allied Health Literature (CINAHL), PsycINFO, Ei Compendex, IEEE Xplore, Applied Science \& Technology Source, Scopus, Web of Science, ClinicalTrials.gov, and Google Scholar. All databases were searched from inception. Proceedings of the Annual Association for Computing Machinery (ACM) Conferences on Human Factors in Computing Systems, the ACM User Interface Software and Technology Symposium, and the IEEE International Symposium on Wearable Computers were searched in the ACM Digital Library from 2012 to 2016. Initial searches were conducted in February 2016 and updates were performed in May 2017. Bibliographies of relevant studies were also reviewed for additional references. A hand search of JMIR Publications journals was completed in July 2017. It should be noted that in the biomedical literature this type of separate search would be classified as a search of the "grey literature"; however, in the fields of computer science, engineering, and human computer interaction, conference proceedings are considered the primary source of scientific literature [30].

The complete search strategies for each database are reported in Multimedia Appendix 1. Database-specific subject headings and keyword variants for each of the two main concepts-dementia/cognitive impairment and mobile technology — were identified and combined. Results were limited to the English language, and animal studies were excluded.

\section{Study Eligibility}

Only empirical studies were included in this systematic review. Inclusion criteria required the study to use a mHealth app on a tablet, a mobile phone, a personal digital assistant, another handheld mHealth device, or a mHealth app accessed from a computer as an intervention for persons with MCI, Alzheimer disease, or dementia. Studies using computers were only included if they accessed a program that was also a mHealth app.

Inclusion criteria required there be at least one quantitative health outcome in the study. Persons aged 18 years or younger were not included because the focus of this study was on cognitive impairment that develops during adulthood. Case series of more than two subjects, case-control studies, cross-sectional studies, and cohort studies were included. Studies were excluded if (1) the study focused primarily on participant populations outside of those with MCI, Alzheimer disease, and dementia. This included populations with diagnoses of traumatic brain injury, human immunodeficiency virus, multiple sclerosis, serious mental illness, intellectual disabilities, or active status as a caregiver, or (2) the primary purpose of the mHealth app was to screen for illness, make an assessment, or determine diagnosis. These studies were excluded because the focus of this study was on active mHealth interventions. Caregivers were not a target of this review. However, some studies included both persons with cognitive impairment and their caregivers. Studies were ruled out if caregivers were the population focus of the study. 


\section{Study Selection and Data Extraction}

Screening of records by title and abstract were completed independently by two authors (DB and BS). An adjudication process was used by the two authors, where they met face-to-face to review screened records. When the authors did not agree on a record, they came to a consensus together through discussion and re-review of the record. The same process was used when evaluating full articles for inclusion and when categorizing the final included articles with the Oxford Centre for Evidence-based Medicine (OCEBM) Levels of Evidence system. Both reviewers independently reviewed full articles and completed data extraction. Using a standard approach, they extracted study design, intervention type, technology type, population diagnoses, mean age of population, mean Mini-Mental Status Examination (MMSE) or comparable cognitive exam, health outcomes, and information on the effectiveness of the mHealth app. Study quality was assessed and categorized using the OCEBM Levels of Evidence System, where studies are categorized into one of five levels of evidence, with one being the strongest level [31]. Levels of evidence using the OCEBM system are (1) level 1: systematic reviews of randomized controlled trials (RCTs), individual RCTs, and all-or-none case series; (2) level 2: systematic reviews of cohort studies, individual cohort studies, and "outcomes" research; (3) level 3: systematic review of case-control studies and individual case-control studies; (4) level 4: case-series and poor quality cohort studies; and (5) level 5: expert opinion. Recommendation grades are listed as consistent level 1 studies ("A"), consistent level 2 or 3 studies or extrapolations from level 1 studies ("B"), level 4 studies or extrapolations from level 2 or 3 studies ("C"), and level 5 evidence or troubling inconsistent or inconclusive studies of any level (“D”) [31].

\section{Results}

A total of 4752 records were identified through database searches. After removing duplicates, 3955 unique titles and abstracts were screened, and 476 full articles were reviewed (Figure 1) [32]. Division of these records according to database can be found in Table 1. A total of 24 separate articles met study inclusion criteria.

Figure 1. PRISMA flow diagram.

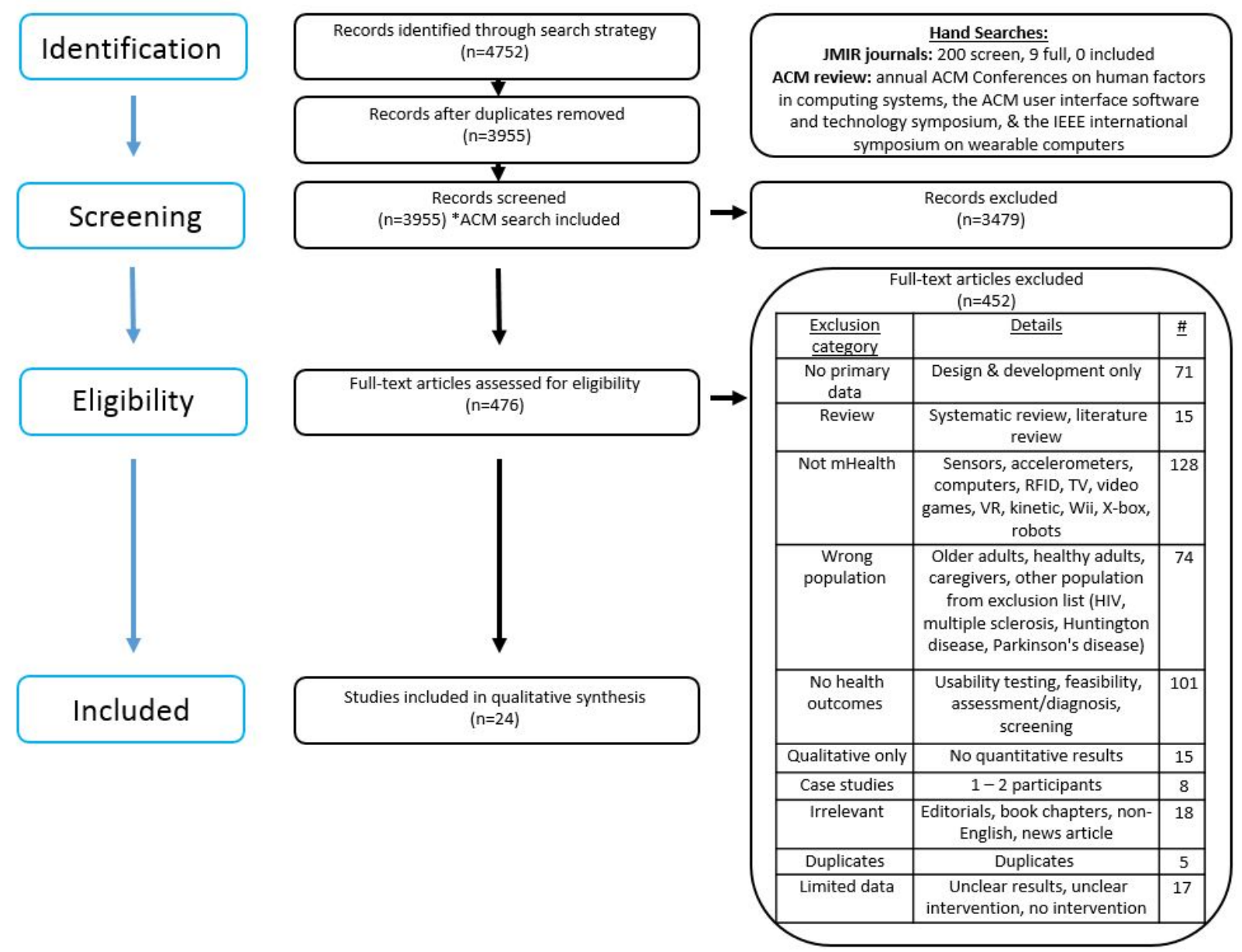


Table 1. Search results with and without duplicates.

\begin{tabular}{lll}
\hline Database & Duplicates included, $\mathrm{n}$ & Duplicates removed, $\mathrm{n}$ \\
\hline ACM Digital Library & 292 & 289 \\
Applied Science \&Technology Source & 37 & 23 \\
CINAHL & 360 & 291 \\
ClinicalTrials.gov & 47 & 47 \\
Cochrane Library & 224 & 105 \\
Ei Compendex & 120 & 106 \\
EMBASE & 736 & 640 \\
Google Scholar & 67 & 37 \\
IEEE Xplore & 453 & 344 \\
Ovid MEDLINE & 1277 & 1276 \\
PsycINFO & 250 & 134 \\
PubMed & 507 & 507 \\
Scopus & 207 & 106 \\
Web of Science & 175 & 50 \\
Total & 4752 & 3955 \\
\hline
\end{tabular}

Health outcomes were extracted and grouped by intervention type (Table 2). Of the 24 individual studies included, 14 studies lacked controls. The majority of studies were small in size. Using the Modified OCEBM Levels of Evidence rating system, four studies met criteria for level 2 evidence [33-36] and none met criteria for either level 1 or level 3 evidence.
The remaining $83 \%$ (20/24) studies were identified as meeting criteria for level 4 evidence (Tables 3-6). All studies were found in biomedical journals or were from biomedical conferences. None of the included studies came from the engineering literature. Two of the four level 2 studies showed some evidence of efficacy $[33,39]$. When looking at all 24 studies regardless of quality, 58\% (14/24) showed some degree of efficacy.

Table 2. Health outcomes and efficacy by mHealth intervention type $(\mathrm{N}=24)$.

\begin{tabular}{|c|c|c|c|}
\hline Intervention type & Studies, $\mathrm{n}^{\mathrm{a}}$ & Health outcomes & $\begin{array}{l}\text { Studies with } \\
\text { efficacy, n (\%) }\end{array}$ \\
\hline Cognitive training with no games & 6 & Cognition [33-35,37-39]; function [35]; mood [33] & $5(83)$ \\
\hline Serious games & 4 & Cognition [36,40-42]; mood, anxiety, stress [36] & $1(25)$ \\
\hline Wandering and wayfinding & 1 & Cognition [43]; unsafe walking behavior [43] & $1(100)$ \\
\hline Reminiscence therapy & 2 & $\begin{array}{l}\text { Cognition [19]; communication ability [19]; mood [19]; social interest } \\
\text { [19]; psychological stability [44] }\end{array}$ & $2(100)$ \\
\hline Prompts and multicomponent interventions & 4 & $\begin{array}{l}\text { Cognition [20,45]; subjective report of cognition [45]; mood [45]; psy- } \\
\text { chological stability [44]; perceived autonomy [20,46]; feeling of compe- } \\
\text { tence [20]; number of caregiver and patient unmet needs [20]; quality } \\
\text { of life for caregiver or patient }[20,45,46] \text {; caregiver burden [45] }\end{array}$ & $1(25)$ \\
\hline Engagement interventions & 7 & $\begin{array}{l}\text { Cognition [47]; well-being and mood [47-49]; behavioral and psycholog- } \\
\text { ical symptoms of dementia [50-52]; engagement in activities [47,48]; } \\
\text { quality of life of patient [48]; helpfulness to caregiver [53] }\end{array}$ & $5(71)$ \\
\hline Exercise intervention & 1 & $\begin{array}{l}\text { Quality of life, self-efficacy, change in weekly steps taken, 6-min walk, } \\
\text { Mini-Physical Performance Test [54] }\end{array}$ & $0(0)$ \\
\hline Total & 24 & & $14(58)$ \\
\hline
\end{tabular}

${ }^{\mathrm{a} C a t e g o r i e s ~ a r e ~ n o t ~ m u t u a l l y ~ e x c l u s i v e ; ~ o n e ~ a r t i c l e ~ w a s ~ c o u n t e d ~ t w i c e . ~}$ 
Table 3. Cognitive training interventions $(n=6)$.

\begin{tabular}{|c|c|c|c|c|c|c|c|}
\hline Author, year & Study type & $\begin{array}{l}\text { Intervention } \\
\text { type }\end{array}$ & $\begin{array}{l}\text { Technology } \\
\text { type }\end{array}$ & Population $^{\mathrm{a}}$ & Outcomes & $\begin{array}{l}\text { OCEBM } \\
\text { level }^{\text {b }}\end{array}$ & Effective/Results ${ }^{c}$ \\
\hline Barnes et al, 2006 [33] & RCT & $\begin{array}{l}\text { Cognitive } \\
\text { training }\end{array}$ & Computer & $\begin{array}{l}36 \text { pts w/ MCI; age: } \\
\text { mean } 74 \text { years; } \\
\text { RBANS total: mean } \\
86.6\end{array}$ & $\begin{array}{l}\text { Cognition, } \\
\text { mood }\end{array}$ & 2 & $\begin{array}{l}\text { No improvement in } \\
\text { cognition (RBANS } \\
\text { total) }\end{array}$ \\
\hline Chan et al, 2017 [34] & $\begin{array}{l}\text { RCT-single } \\
\text { blind }\end{array}$ & $\begin{array}{l}\text { Cognitive } \\
\text { training }\end{array}$ & Tablet & $\begin{array}{l}99 \mathrm{pts} \text { w/ MCI; age: } \\
\text { mean } 68.7 \text { years; } \\
\text { MOCA: mean } 24.4\end{array}$ & Cognition & 2 & $\begin{array}{l}\text { Yes, treatment group } \\
\text { had improvements in } \\
\text { working memory; } \\
\text { both treatment and } \\
\text { control groups had } \\
\text { improvements in im- } \\
\text { mediate and delayed } \\
\text { recall }\end{array}$ \\
\hline Gooding et al, 2015 [37] & RCT & $\begin{array}{l}\text { Cognitive } \\
\text { training }\end{array}$ & Computer & $\begin{array}{l}74 \text { pts w/ subclinical } \\
\text { cognitive decline; } \\
\text { age: mean } 75.6 \\
\text { years; mMMSE: } \\
\text { mean } 50.6\end{array}$ & Cognition & 4 & $\begin{array}{l}\text { Yes, improvement in } \\
\text { cognition (mMMSE, } \\
\text { BSRT, and LMS) }\end{array}$ \\
\hline Han et al, 2014 [38] & Pilot & $\begin{array}{l}\text { Cognitive } \\
\text { training }\end{array}$ & Tablet & $\begin{array}{l}10 \text { pts w/ MCI; age } \\
\text { mean: } 69.7 \text { years; } \\
\text { MMSE: mean } 26.7 \text {; } \\
\text { CDR: mean } 0.5\end{array}$ & Cognition & 4 & $\begin{array}{l}\text { Yes, significant im- } \\
\text { provement in cogni- } \\
\text { tion (word list memo- } \\
\text { ry test) }\end{array}$ \\
\hline Mansbach et al, 2017 [39] & $\begin{array}{l}\text { Controlled } \\
\text { trial }\end{array}$ & $\begin{array}{l}\text { Cognitive } \\
\text { training }\end{array}$ & $\begin{array}{l}\text { Mobile app } \\
\text { from computer }\end{array}$ & $\begin{array}{l}38 \text { pts w/ normal } \\
\text { cognition, MCI, and } \\
\text { mild dementia; age: } \\
\text { mean } 78.1 \text { years; } \\
\text { BCAT: mean } 37.3\end{array}$ & $\begin{array}{l}\text { Cognition, } \\
\text { subjective } \\
\text { report of } \\
\text { cognition }\end{array}$ & 4 & $\begin{array}{l}\text { Yes, treatment group } \\
\text { had greater improve- } \\
\text { ments in cognition }\end{array}$ \\
\hline Tarraga et al, 2006 [35] & RCT & $\begin{array}{l}\text { Cognitive } \\
\text { training }\end{array}$ & Computer & $\begin{array}{l}43 \text { pts w/ MCI; age: } \\
\text { mean } 76.7 \text { years; } \\
\text { MMSE: mean } 21.9\end{array}$ & $\begin{array}{l}\text { Cognition, } \\
\text { function }\end{array}$ & 2 & $\begin{array}{l}\text { Yes, improvement in } \\
\text { cognition (ADAS- } \\
\text { Cog, MMSE); no } \\
\text { functional improve- } \\
\text { ments }\end{array}$ \\
\hline
\end{tabular}

a BCAT: Brief Cognitive Assessment Tool; CDR: Clinical Dementia Rating scale; MCI: mild cognitive impairment: MMSE: Mini-Mental State Examination; mMMSE: Modified Mini-Mental State Examination; MOCA: Montreal Cognitive Assessment.

${ }^{\mathrm{b}}$ Oxford Centre for Evidence-based Medicine's Levels of Evidence and Grades of Recommendation (1=highest quality; 5=lowest quality).

${ }^{\mathrm{c}}$ ADAS-Cog: Alzheimer's disease Assessment Scale cognitive subscale; BSRT: Buschke Selective Reminding Test; LMS: Logical Memory Subtest; RBANS: Repeatable Battery for the Assessment of Neuropsychological Status. 
Table 4. Serious games with cognitive training $(n=4)$.

\begin{tabular}{|c|c|c|c|c|c|c|c|}
\hline Author, year & $\begin{array}{l}\text { Study } \\
\text { type }\end{array}$ & $\begin{array}{l}\text { Intervention } \\
\text { type }\end{array}$ & $\begin{array}{l}\text { Technology } \\
\text { type }\end{array}$ & Population $^{\mathrm{a}}$ & Outcomes & $\begin{array}{l}\text { OCEBM } \\
\text { level }^{\text {b }}\end{array}$ & Effective/Results \\
\hline Finn and McDonald, 2011 [36] & RCT & $\begin{array}{l}\text { Serious games, } \\
\text { cognitive train- } \\
\text { ing }\end{array}$ & Computer & $\begin{array}{l}25 \text { pts w/ MCI; age: } \\
\text { mean } 74.2 \text { years; } \\
\text { MMSE: mean } 27.8\end{array}$ & $\begin{array}{l}\text { Cognition, } \\
\text { Mood, Anxi- } \\
\text { ety, Stress }\end{array}$ & 2 & $\begin{array}{l}\text { No, only improvement } \\
\text { was in visual sus- } \\
\text { tained attention; no } \\
\text { improvement in cogni- } \\
\text { tion, depression, or } \\
\text { anxiety }\end{array}$ \\
\hline Hsiung et al, 2009 [40] & Pilot & $\begin{array}{l}\text { Serious games, } \\
\text { cognitive train- } \\
\text { ing }\end{array}$ & $\begin{array}{l}\text { Handheld } \\
\text { device }\end{array}$ & $\begin{array}{l}17 \text { pts total } 12 \mathrm{w} / \\
\text { MCI, } 2 \text { healthy, } 3 \mathrm{w} / \\
\text { subjective memory } \\
\text { complaints; age: } \\
\text { mean } 72 \text { years: } \\
\text { MMSE: mean NR }\end{array}$ & Cognition & 4 & $\begin{array}{l}\text { No improvement in } \\
\text { cognition }\end{array}$ \\
\hline Manera et al, 2015 [41] & Pilot & $\begin{array}{l}\text { Serious games, } \\
\text { cognitive train- } \\
\text { ing }\end{array}$ & Tablet & $\begin{array}{l}9 \text { pts w/ MCI, } 12 \text { pts } \\
\text { w/ Alzheimer dis- } \\
\text { ease; age mean } 78.4 \\
\text { years; MMSE MCI: } \\
\text { mean } 27.2 \text {, MMSE } \\
\text { Alzheimer disease: } \\
\text { mean } 18.4\end{array}$ & Cognition & 4 & $\begin{array}{l}\text { Yes, improvement in } \\
\text { praxis \& executive } \\
\text { function }\end{array}$ \\
\hline Merilampi et al, 2014 [42] & Pilot & $\begin{array}{l}\text { Serious games, } \\
\text { cognitive train- } \\
\text { ing }\end{array}$ & $\begin{array}{l}\text { Tablet, com- } \\
\text { puter }\end{array}$ & $\begin{array}{l}16 \text { pts w/ mild-to- } \\
\text { moderate cognitive } \\
\text { impairment; age: } \\
\text { mean } 90 \text { years; } \\
\text { MMSE: mean } 21.6\end{array}$ & Cognition & 4 & $\begin{array}{l}\text { No improvement in } \\
\text { cognition }\end{array}$ \\
\hline
\end{tabular}

${ }^{\mathrm{a}}$ MCI: mild cognitive impairment; MMSE: Mini-Mental State Examination; NR: not reported.

${ }^{\mathrm{b}}$ Oxford Centre for Evidence-based Medicine's Levels of Evidence and Grades of Recommendation (1=highest quality; 5=lowest quality). 
Table 5. Wandering and wayfinding $(n=1)$, reminiscence therapy $(n=2)$, and prompts and multicomponent ( $=4)$ interventions.

\begin{tabular}{|c|c|c|c|c|c|c|c|}
\hline Author, year & $\begin{array}{l}\text { Study } \\
\text { type }\end{array}$ & $\begin{array}{l}\text { Intervention } \\
\text { type }\end{array}$ & $\begin{array}{l}\text { Technology } \\
\text { type }\end{array}$ & Population $^{\mathrm{a}}$ & Outcomes $^{b}$ & $\begin{array}{l}\text { OCEBM } \\
\text { level }^{\mathrm{c}}\end{array}$ & Effective/Results ${ }^{b}$ \\
\hline Hettinga et al, 2009 [43] & Pilot & $\begin{array}{l}\text { Wandering and } \\
\text { wayfinding }\end{array}$ & PDA & $\begin{array}{l}4 \text { pts w/ mild demen- } \\
\text { tia; age: } \geq 55 \text { years; } \\
\text { MMSE: range } 17-25\end{array}$ & $\begin{array}{l}\text { Unsafe walking } \\
\text { behaviors, } \\
\text { working memo- } \\
\text { ry }\end{array}$ & 4 & $\begin{array}{l}\text { No unsafe walking } \\
\text { behaviors }\end{array}$ \\
\hline Hattink et al, 2016 [20] & $\mathrm{RCT}$ & $\begin{array}{l}\text { Multicompo- } \\
\text { nent interven- } \\
\text { tion }\end{array}$ & $\begin{array}{l}\text { Early detection } \\
\text { system-touch- } \\
\text { screen or mo- } \\
\text { bile device }\end{array}$ & $\begin{array}{l}42 \text { pts w/ MCI and } \\
\text { dementia; age: mean } \\
78.7 \text { years; MMSE: } \\
\text { mean } 18.1\end{array}$ & $\begin{array}{l}\text { Cognition, } \\
\text { QOL-AD for } \\
\text { CG or patient, } \\
\text { perceived auton- } \\
\text { omy, feeling of } \\
\text { competence, } \\
\text { number of CG } \\
\text { and patient un- } \\
\text { met needs }\end{array}$ & 4 & $\begin{array}{l}\text { No differences in } \\
\text { QOL-AD for CG or } \\
\text { patient, perceived au- } \\
\text { tonomy, grade for } \\
\text { QOL, feeling of com- } \\
\text { petence, MMSE, } \\
\text { number of caregiver } \\
\text { and patient unmet } \\
\text { needs. }\end{array}$ \\
\hline Imbeault et al, 2016 [45] & $\begin{array}{l}\text { Case } \\
\text { series }\end{array}$ & Prompts & $\begin{array}{l}\text { Mobile phone } \\
\text { app }\end{array}$ & $\begin{array}{l}3 \text { pts w/ Alzheimer } \\
\text { disease; age: mean } \\
69 \text { years; MMSE: } \\
\text { mean } 28\end{array}$ & $\begin{array}{l}\text { Cognition, sub- } \\
\text { jective report of } \\
\text { cognition, de- } \\
\text { pression, CG } \\
\text { burden }\end{array}$ & 4 & $\begin{array}{l}\text { No, almost all cogni- } \\
\text { tive tests remained the } \\
\text { same or decreased. } \\
\text { Unclear results for de- } \\
\text { pression and caregiver } \\
\text { burden. }\end{array}$ \\
\hline Meiland et al, 2012 [46] & Pilot & Prompts & $\begin{array}{l}\text { Mobile device, } \\
\text { sensors, touch- } \\
\text { screen, \& actua- } \\
\text { tors }\end{array}$ & $\begin{array}{l}12 \text { pts w/ MCI, de- } \\
\text { mentia, or } \\
\text { Alzheimer disease; } \\
\text { age: range } 57-84 \\
\text { years; MMSE: range } \\
\text { 17-25 (mean age and } \\
\text { MMSE NR) }\end{array}$ & $\begin{array}{l}\text { Quality of life, } \\
\text { perceived auton- } \\
\text { omy }\end{array}$ & 4 & $\begin{array}{l}\text { No effect on QOL of } \\
\text { Patient or CG, or per- } \\
\text { ceived autonomy. }\end{array}$ \\
\hline Yasuda et al, 2013 [44] & Pilot & $\begin{array}{l}\text { Prompts and } \\
\text { reminiscence } \\
\text { therapy }\end{array}$ & Computer & $\begin{array}{l}4 \text { pts w/ Alzheimer } \\
\text { disease; age: mean } \\
78.7 \text { years; MMSE: } \\
\text { mean } 19.5\end{array}$ & $\begin{array}{l}\text { Psychological } \\
\text { stability, com- } \\
\text { munication abil- } \\
\text { ity, IADL com- } \\
\text { pletion }\end{array}$ & 4 & $\begin{array}{l}\text { Yes, } 3 \text { of } 4 \text { partici- } \\
\text { pants had benefit in } \\
\text { psychological stabili- } \\
\text { ty. Improvements } \\
\text { were also noted in } \\
\text { communication ability } \\
\text { and in IADL competi- } \\
\text { tion. }\end{array}$ \\
\hline O'Rourke et al, 2011 ${ }^{\mathrm{d}}$ [19] & Pilot & $\begin{array}{l}\text { Reminiscence } \\
\text { therapy }\end{array}$ & $\begin{array}{l}\text { Mobile app } \\
\text { from computer }\end{array}$ & $\begin{array}{l}6 \text { pts w/ dementia; } \\
\text { age: mean } 72 \text { years; } \\
\text { MMSE: mean } 17.8\end{array}$ & $\begin{array}{l}\text { Cognition, com- } \\
\text { munication abil- } \\
\text { ity (FLCI), de- } \\
\text { pression, social } \\
\text { interest ques- } \\
\text { tionnaire }\end{array}$ & 4 & $\begin{array}{l}\text { Yes, MMSE scores } \\
\text { improved in half of } \\
\text { pts FLCI scores im- } \\
\text { proved or remained } \\
\text { stable in all but one } \\
\text { participant. }\end{array}$ \\
\hline
\end{tabular}

${ }^{a}$ MCI: mild cognitive impairment; MMSE: Mini-Mental State Examination; NR: not reported.

${ }^{\mathrm{b}}$ CG: caregiver; FLCI: Functional Linguistic Communication Inventory; IADL: Instrumental Activities of Daily Living; QOL-AD: Quality of Life Scale in Alzheimer's Disease.

'Oxford Centre for Evidence-based Medicine's Levels of Evidence and Grades of Recommendation (1=highest quality; 5=lowest quality).

${ }^{\mathrm{d}}$ Categories are not mutually exclusive; one article was counted in both the reminiscence therapy and prompts section. 
Table 6. Engagement $(n=7)$ and exercise $(n=1)$ interventions.

\begin{tabular}{|c|c|c|c|c|c|c|c|}
\hline Author, year & Study type & $\begin{array}{l}\text { Intervention } \\
\text { type }\end{array}$ & $\begin{array}{l}\text { Technology } \\
\text { type }\end{array}$ & Population $^{\mathrm{a}}$ & Outcomes $^{b}$ & $\begin{array}{l}\text { OCEBM } \\
\text { level }^{\mathrm{c}}\end{array}$ & Effective/Results \\
\hline Astell et al, 2016 [49] & $\begin{array}{l}\text { Controlled } \\
\text { trial }\end{array}$ & Engagement & Tablet & $\begin{array}{l}30 \text { pts w/ dementia; } \\
\text { age: mean } 87.3 \\
\text { years; MOCA: mean } \\
13.4\end{array}$ & Enjoyment & 4 & $\begin{array}{l}\text { Yes, } 88 \% \text { of pa- } \\
\text { tients reportedly } \\
\text { enjoyed the games }\end{array}$ \\
\hline Hsu et al, 2016 [50] & Case series & Engagement & Tablet & $\begin{array}{l}3 \text { pts w/ dementia; } \\
\text { age: mean } 78 \text { years; } \\
\text { MOCA: mean } 23.5 \\
\text { (1 pt refused MO- } \\
\text { CA) }\end{array}$ & $\begin{array}{l}\text { Behavioral } \\
\text { and psycho- } \\
\text { logical } \\
\text { symptoms of } \\
\text { dementia }\end{array}$ & 4 & $\begin{array}{l}\text { Yes, decreased use } \\
\text { of "as-needed" } \\
\text { medications for be- } \\
\text { havioral problems }\end{array}$ \\
\hline Leng et al, 2014 [47] & Pilot & Engagement & Tablet & $\begin{array}{l}6 \text { pts w/ dementia; } \\
\text { age: mean } 77 \text { years; } \\
\text { MMSE: mean } 21\end{array}$ & $\begin{array}{l}\text { Cognition, } \\
\text { mood, en- } \\
\text { gagement, } \\
\text { well-being }\end{array}$ & 4 & $\begin{array}{l}\text { Yes, tablet activi- } \\
\text { ties at least an } \\
\text { equal positive ef- } \\
\text { fect on mood, en- } \\
\text { gagement, and } \\
\text { well-being vs tradi- } \\
\text { tional group activi- } \\
\text { ties }\end{array}$ \\
\hline Lim et al, 2013 [53] & Pilot & Engagement & Tablet & $\begin{array}{l}21 \text { dyads of people } \\
\text { with early dementia } \\
\text { and CGs; PWD age: } \\
\text { mean } 73.5 \text { years; } \\
\text { MMSE: NR }\end{array}$ & $\begin{array}{l}\text { Helpfulness } \\
\text { to caregiver }\end{array}$ & 4 & $\begin{array}{l}\text { Yes, } 47.6 \% \text { of } \mathrm{CG} \\
\text { found tablet some- } \\
\text { what, moderately, } \\
\text { or extremely help- } \\
\text { ful }\end{array}$ \\
\hline Tyack et al, 2015 [48] & Pilot & Engagement & Tablet & $\begin{array}{l}12 \text { dyads-PWD and } \\
\text { CGs; age: mean } 75 \\
\text { years; MMSE: NR }\end{array}$ & $\begin{array}{l}\text { QOL-AD; } \\
\text { Visual Ana- } \\
\text { logue Scale } \\
\text { for Happi- } \\
\text { ness, Well- } \\
\text { ness, and in- } \\
\text { terestedness }\end{array}$ & 4 & $\begin{array}{l}\text { No improvement in } \\
\text { happiness, well- } \\
\text { ness, interestedness }\end{array}$ \\
\hline Vahia et al, 2016 [51] & $\begin{array}{l}\text { Open-label } \\
\text { study }\end{array}$ & Engagement & Tablet & $\begin{array}{l}36 \text { pts w/ dementia; } \\
\text { age: mean } 79.9 \\
\text { years; MMSE: NR }\end{array}$ & $\begin{array}{l}\text { Behavioral } \\
\text { and psycho- } \\
\text { logical } \\
\text { symptoms of } \\
\text { dementia, } \\
\text { agitation }\end{array}$ & 4 & $\begin{array}{l}\text { Yes, agitation de- } \\
\text { creased post inter- } \\
\text { vention }\end{array}$ \\
\hline Van Der Ploeg et al, 2015 [52] & RCT & Engagement & $\begin{array}{l}\text { Mobile app } \\
\text { from computer }\end{array}$ & $\begin{array}{l}17 \text { pts w/ dementia; } \\
\text { age: mean } 86.7 \\
\text { years; MMSE: mean } \\
7.3\end{array}$ & $\begin{array}{l}\text { Behavioral } \\
\text { and psycho- } \\
\text { logical } \\
\text { symptoms of } \\
\text { dementia, } \\
\text { agitation }\end{array}$ & 4 & $\begin{array}{l}\text { No significant re- } \\
\text { duction in agitation }\end{array}$ \\
\hline Vidoni et al, 2016 [54] & Pilot & Exercise & $\begin{array}{l}\text { Accelerometer, } \\
\text { mobile app } \\
\text { from computer }\end{array}$ & $\begin{array}{l}21 \text { pts as normal } \\
\text { control, } 9 \text { pt w/ } \\
\text { Alzheimer disease; } \\
\text { control age: mean } \\
72.3 \text { years, } \\
\text { Alzheimer disease } \\
\text { age: mean } 69.6 \\
\text { years, MMSE: NR }\end{array}$ & $\begin{array}{l}\text { QOL, self- } \\
\text { efficacy, } \\
\text { change in } \\
\text { weekly steps } \\
\text { taken, 6-min } \\
\text { walk, Mini- } \\
\text { Physical Per- } \\
\text { formance } \\
\text { Test }\end{array}$ & 4 & $\begin{array}{l}\text { No improvement in } \\
\text { outcomes }\end{array}$ \\
\hline
\end{tabular}

${ }^{\mathrm{a} C G}$ : caregiver; MMSE: Mini-Mental State Examination; MOCA: Montreal Cognitive Assessment; NR: not reported; PWD: people with dementia. ${ }^{b}$ QOL: Quality of Life; QOL-AD: Quality of Life Scale in Alzheimer's Disease.

'Oxford Centre for Evidence-based Medicine's Levels of Evidence and Grades of Recommendation (1=highest quality; 5=lowest quality). 


\section{Cognitive Training and Serious Games}

\section{Study Characteristics}

Cognitive training with and without serious games made up a large number of the studies in this review $(n=10)$. Intervention design and duration varied significantly among studies. All studies in the two groups incorporated cognition as an outcomes measure, another included function [35] and still others used mood, stress, and anxiety $[33,36]$ as outcomes. When examining the two groups in combination $60 \%(6 / 10)$ of the studies showed some degree of efficacy [34,35,37-39,41] (see Tables 3 and 4). All four studies in this review that met criteria for level 2 quality of evidence fell into one of these two categories [33-36].

\section{Description of Apps and Technology}

A number of studies in this group used commercially available cognitive training programs, such as those by the company Lumos Laboratory, marketed as Lumosity, and by the company Posit Science marketed as BrainHQ. Other studies reported on mHealth apps that had been developed through research. Many of these studies were conducted on computers; however, studies were only included if the apps were able to be accessed by mobile devices. One study that demonstrated efficacy used a tablet-based Chinese calligraphy program as a form of cognitive training [34].

\section{Wayfinding and Wandering \\ Study Characteristics}

Although a number of pilot studies reported on experiments involving navigation systems for persons with dementia, only one met criteria for inclusion [43]. Health outcomes for the study consisted of working memory and unsafe walking behaviors [43]. The study proved to be effective in that no unsafe walking behaviors were found for those who used the navigation system.

\section{Description of Apps and Technology}

In the study by Hettinga et al [43], software called TomTom was used for navigation support. They studied the safety of TomTom use by people with dementia and the effectiveness of familiar versus unfamiliar voice prompts. The use of navigation software was found to be safe based on observations of street-crossing behavior, response to navigation instructions, and number of occurrences of stopping at device prompts. Additionally, they observed that familiar voice prompts were more effective compared to unfamiliar ones. This was determined by measuring walking time, number of errors (route deviations and repeated instructions), and number of times assistance was requested. Warning sounds seemed to have a negative effect on wayfinding [43]. Participants were captured on video. These videos were coded for unsafe walking behaviors [43].

\section{Reminiscence Therapy}

\section{Study Characteristics}

Two studies incorporated a reminiscence therapy intervention $[19,44]$. Health outcomes included cognition, communication ability, mood, social interest, and psychological stability. Both studies showed some degree of efficacy [19].

\section{Description of Apps and Technology}

O'Rourke et al [19] used the Web-based video website YouTube to help facilitate reminiscence therapy in persons with dementia. Five of six participants showed improvement or stability in their communication ability over the 6-week pilot study. They concluded that the website YouTube is a suitable tool for delivering personalized computer-based reminiscence therapy [19]. YouTube also functions as a mobile app.

In their pilot study, Yasuda et al [44] used a videophone as both a remote reminiscence conversation system and as a schedule prompter system. The remote reminiscence conversation system shared reminiscence photos through a videophone triggered by the conversation partner on the other end of the phone. The study results showed following conversations with the system patients had improved psychological stability, verbal communication, and rates of instrumental ADL completion. The schedule prompter system used more than 10 different video reminders, such as prompts to take medications or to prepare meals. Individual experiments were conducted to evaluate each system using four patients with dementia. The first experiment evaluated the effectiveness of the remote reminiscence conversation system by performing two tasks: watching TV and remote video chatting. Results were measured using the Gottfries-Brane-Steen (GBS) scale that measures psychological variables such as confusion, irritability, anxiety, restlessness, reduced mood, and agony. The GBS scale is scored on a scale from zero (most stable) to six (least stable). Results showed that three of four patients obtained psychological stability as defined by the authors. The second experiment determined the effectiveness of a schedule prompter system in completing the scheduled task. Participants received three different types of video prompts: navigational prompts to move toward the computer, motivational prompts to inspire completion of tasks, and scheduled prompts to remind participants of tasks scheduled for completion. The results described the mean completion of tasks for the four patients to as $83 \%$ while using the prompter system [44].

\section{Prompts and Multicomponent Interventions}

\section{Study Characteristics}

We found four studies in these combined categories [20,44-46]. Health outcomes for this group included cognition, subjective report of cognition, mood, psychological stability, perceived autonomy, feeling of competence, the number of caregiver and patient unmet needs, caregiver burden, and the quality of life for the caregiver and patient. Of the four studies in this category, only the study by Yasuda et al [44] showed improvement in health outcomes [44].

\section{Description of Apps and Technology}

Yasuda et al [44] was previously described in the reminiscence therapy section because the study contained both reminiscence therapy and prompt components. Overall, in these studies, prompts were delivered in either an auditory or visual manner. The use of mobile devices in this group varied. Imbeault et al 
[45] studied the impact of an electronic organizer "AP@LZ" on the cognition, subjective report of cognition, depression, and caregiver burden of three persons with Alzheimer disease. Unfortunately, there were no cognitive benefits. The results of the effect on depression and caregiver burden were unclear [45].

In 2009, Meiland et al [46] evaluated the use of a digital prosthetic, "COGNOW Day Navigator," by 12 persons with dementia and their caregivers. The digital prosthetic was designed to help with memory, social contacts, daily activities, and safety. Participants rated the study as useful and user-friendly. Effectiveness of the system could not be determined because of the short study duration and instability of the digital prosthetic prototype.

Lastly, one multicomponent intervention study by Hattink et al [20] met criteria for inclusion. This intervention, "Rosetta," targeted four domains of required support for persons with dementia: (1) prompts and reminders, (2) leisure, (3) communication, and (4) safety. The Rosetta intervention was tested with 42 patients with either MCI or Alzheimer disease. Contained in their measured health outcomes were cognition, perceived autonomy, feelings of competence, the number of unmet caregiver and patient needs, and quality of life of the caregiver and patient. No improvements in outcomes occurred following the intervention [20].

\section{Engagement Interventions}

\section{Study Characteristics}

Past studies demonstrated that recreational activities and engagement can lead to persons with dementia having more positive affect, decreased agitation, and decreased passivity $[22,23]$. Health outcomes varied by study. They consisted of mood, engagement, well-being, behavioral and psychological symptoms of dementia, agitation, and quality of life of the caregiver and patient. Five of the seven $(71 \%)$ studies using a mHealth app intervention to engage people with dementia in activities had evidence of efficacy [47-53].

\section{Description of Apps and Technology}

Tablets were the technology of choice for these studies. In an adult day program, Leng et al [47] studied how iPad group activity sessions compared to traditional group engagement activities of cooking and arts and crafts for persons with dementia. The study found that for the persons with dementia who participated, iPad activities had at least an equal positive effect on mood, engagement, and well-being as compared to traditional group activities [47].

Another group, Lim et al [53], studied the usability of iPads by persons with dementia and their caregivers. In all, 95\% of persons with dementia participating in the study had not previously used an iPad. Apps in the categories of art, music, simple interactive games, and relaxation were loaded onto each iPad. The patient-caregiver dyad was given the iPad to take home and use for 7 days with the recommendation that the caregiver provide 30 minutes of daily supervision and interaction while the person with dementia used the iPad. Nearly half of the caregivers indicated the iPad was somewhat, moderately, or extremely helpful.
Astell et al [49] studied 30 persons with dementia and measured the impact familiar and nonfamiliar games on a tablet had on the participants' enjoyment. A total of $90 \%$ of participants attempted to use the tablet. Regardless of familiarity, close to $90 \%$ of participants displayed enjoyment from playing games on a tablet.

Tyack et al [48] developed and tested an art viewing tablet app for persons with dementia as a well-being intervention. Twelve patient-caregiver dyads participated. Participants were asked to use the tablet and program five times over a 2-week span and were given a list of questions to facilitate conversation while using the app. There were no significant pre-post differences in any of the outcomes measured, including patient happiness, wellness, engagement, and patient and caregiver quality of life [48]. Despite this, there was a trend toward improvement in all outcome categories, indicating that a larger study might demonstrate effect.

Van der Ploeg et al [52] tested Internet video conferencing (Skype) and telephone calls with family members as an intervention to reduce agitation in persons with dementia. Preintervention and postintervention measurements showed no difference in Cohen-Mansfield Agitation Inventory scores [52].

Vahia et al [51] and Hsu et al [51] both used mobile apps on a tablet to treat symptoms of agitation and behavioral and psychological symptoms of dementia. Vahia et al found that their intervention reduced agitation and that all participants including those with severe dementia were able to use apps on the tablet [51]. In the small study by Hsu et al, those who received the tablet intervention had decreased use of "as-needed" medications to treat behavioral and psychological symptoms of dementia [50].

\section{Exercise Intervention}

\section{Study Characteristics}

The one exercise intervention that met inclusion criteria selected health outcomes of quality of life, self-efficacy, change in weekly steps taken, the 6-minute walk time, and the Mini-Physical Performance Test. None of these outcomes improved with the exercise intervention [54].

\section{Description of App and Technology}

Vidoni et al [54] sought to improve the health of persons with dementia by prescribing physical activity in conjunction with using a wearable mHealth device (Fitbit) in a tertiary medical clinic setting. The wearable device was an accelerometer that measured steps taken and communicated with the mHealth app installed on either a mobile device or computer. The randomized trial included participants with normal cognition and Alzheimer disease-related cognitive impairment. The study was designed to last 16 weeks, but only 8 weeks of data was reported. Normal controls improved on their baseline weekly steps taken, whereas those with cognitive impairment did not. Only $62 \%$ of those with cognitive impairment completed the intervention [54]. 


\section{Discussion}

\section{Theoretical Implications}

To our knowledge, this comprehensive review is the first to examine the efficacy of mHealth app interventions on the health outcomes of persons with MCI, Alzheimer disease, and dementia. We employed state-of-the-art methodology in identifying the relevant literature, rating the quality of studies, and extracting standardized data. Using a broad search strategy, we discovered this literature spread across the research and innovation outlets of multiple disciplines. The level of evidence supporting the use of mHealth app interventions for people with these disorders (MCI, Alzheimer disease, and dementia) was low, as reflected by a grade $\mathrm{C}$ level of evidence using the modified OCEBM rating system. Some degree of efficacy was seen in 58\% (14/24) of all included studies. However, given the limited quality of these studies it is difficult to draw any firm conclusions. Given the potential size of the market for these interventions and their apparent potential for improving the care of persons with cognitive impairment, we uncovered a need not only for more research in this area, but also for greater agreement in study design and consensus on health outcome measures.

Unexpectedly, none of the 24 studies came from the primary computer science literature. We attribute this lack of computer science study selection to the inclusion criteria requiring at least one quantitative health outcome. In our review, we found a number of innovative studies of mHealth app interventions for people with cognitive impairment. In the computer science literature, however, the vast majority of these studies lacked any measure of patient health. This significant finding highlights the potential opportunity and need for collaboration between technology researchers and health care professionals to develop mHealth app interventions that improve the health of individuals with cognitive impairment.

\section{Outcomes}

Aim 1 was to examine and catalog the types of quantitative health outcomes used by mHealth app interventions for persons with MCI, Alzheimer disease, and dementia. We found that 101 of $476(21.2 \%)$ articles fully reviewed were excluded because of a lack of health outcomes. There were also inconsistencies in the health outcomes selected by study investigators. There appears to be a lack of consensus on which health care outcomes should be used to evaluate mHealth app interventions targeting those with cognitive impairment. The most commonly used health outcomes were cognition, function, quality of life, mood and well-being, and behavioral and psychological symptoms of dementia. There exists a greater need for consistency in the health outcomes for persons with cognitive impairment in these studies.

\section{Efficacy}

Aim 2 of this study sought to determine the efficacy of mHealth app interventions focused on persons with cognitive impairment that included at least one quantitative health outcome in the study evaluation. This systematic review found that currently there is little evidence to support the efficacy of most mHealth app interventions improving the health of persons with cognitive impairment. The number of mHealth apps continues to grow [3]. However, there is limited oversight and rigor in the development and testing of these apps and their claims of benefit. Lumos Laboratory, doing business as Lumosity, served as the most recent well-publicized example of a company charged with unproven medical claims about the benefits received from their cognitive training mHealth app. The Federal Trade Commission alleged that the company misled consumers in claiming that their product "delays age-related mental decline and protects against dementia and Alzheimer's disease" [55]. Without rigorous RCTs to prove efficacy of individual mHealth app interventions, mHealth apps run the risk of being the newest "snake oil" to treat dementias and associated disorders. More work needs to be done to determine which apps are effective at improving patient outcomes.

\section{Limitations}

Our review has several limitations. The review topic was broad, which creates challenges in terms of in-depth data synthesis. An adjudication process between two authors (DB and BS) was used to find consensus on article screening, full article review, and categorization with the OCEBM Levels of Evidence System. Interrater reliability or kappa was not calculated and is therefore a limitation. This review's original search strategy did not focus explicitly on cognitive training; therefore, there may have been studies in this area that were not captured in this review. In the field of mHealth and in our review the majority of the studies are of small size and lacked sufficient quality in study design.

\section{Future Directions}

Greater need for clinical trials to test mHealth interventions necessitates involvement with the Food and Drug Administration (FDA), the government agency with the widest jurisdiction over the regulation of mobile health technologies [56]. The FDA issued its most recent nonbinding industry and staff guidance document on "mobile medical applications," in 2013 and updated the document in 2015 [57]. In these reports, The FDA defines mobile medical apps as "a mobile app that meets the definition of device in section 201(h) of the Federal Food, Drug and Cosmetic Act (FD\&C Act); and is either intended (1) to be used as an accessory to a regulated medical device; or (2) to transform a mobile platform into a regulated medical device" [57]. Section 201 (h) describes a medical device as one "...intended for use in the diagnosis of disease or other conditions, or in the cure, mitigation, treatment, or prevention of disease, in man..." or "...intended to affect the structure or any function of the body of man..." This definition includes software or apps on computers, websites, and handheld devices.

The FDA has made clear their intention to apply regulatory oversight to the subset of mobile medical apps that transform a mobile platform into a medical device [56,57]. They indicate that they reserve the right to enforce the guidelines at their discretion and will focus on mobile medical apps that have the potential to cause harm to patients [57].

Outside of efficacy and safety, other concerns exist with mHealth technologies, including privacy risks. In their 2016 JAMA letter, Blenner et al [58] showed that $81 \%$ of available 
Android diabetes apps did not have privacy policies and that $48.4 \%$ of diabetes apps with privacy policies shared user information. In downloading and installing an app, health consumers often inadvertently give apps permission to collect personal information [58]. As noted by Blenner et al, there are currently no federal laws to prevent app companies from selling medical app data to third parties [58,59]. One could imagine the potentially damaging effect that could be incurred if sensitive medical information were to be shared with health or life insurance companies or with a prospective employer.

Although not the focus of this review, we found a large number of studies did not take end users' (persons with dementia or caregivers) input into consideration during the development of mHealth interventions, creating potential mismatch between the proposed solution and the participant needs. This suggests an opportunity for greater emphasis on user-centered design. User-centered design is a philosophy and methodology for designing and evaluating systems based on end-user involvement and a strong understanding of end-user characteristics, goals, tasks, needs, capabilities, and contexts [60]. This approach has the ultimate goal of optimal functioning of the human-machine system [61].

Despite these different concerns and drawbacks, mHealth apps possess great potential to improve the health and outcomes of people with cognitive impairment and offer advantages over traditional psychosocial interventions. With the growing numbers of persons with dementia, limitations of family caregivers, and work force shortage, there is an extraordinary need for engagement and social support of persons with dementia. One could imagine mHealth apps that could engage persons with dementia and help improve their mood through serious games or through a mHealth peer-support program. The aim of these apps would not be to replace caregivers or humans, but rather to allow for engagement when caregivers or others are busy or not immediately available.

The use of mHealth apps offer an opportunity to help persons with MCI, Alzheimer disease, and dementia maintain their independence longer than would be otherwise possible. Apps designed to prompt persons with dementia with necessary tasks such as taking medication, taking out the garbage, or cooking meals, could help persons with dementia complete these essential tasks rather than having to hire a caregiver or move into an assisted living to receive support. Similarly, wayfinding apps could improve persons with dementia's ability to travel safely in their community. And remote monitoring apps could allow caregivers and health care providers to supervise or receive notifications of changes in the health status of the person with dementia.

In addition, mHealth apps could improve measurement accuracy of variables pertaining to the health status of persons with dementia. Through ecological momentary assessment, the repeated sampling of a participant's experience or behaviors in real time, an app could provide up-to-date information on a person's mood status or neuropsychiatric symptoms of dementia [62], thus avoiding the pitfalls of recall bias seen with more traditional office-based questionnaires. Monitoring or prompting apps could help measure real-time decline in a person with dementia's cognition or function, offering providers a more accurate depiction of disease progression.

Virtual coaches hold promise as the next generation of mHealth apps and have the potential to help persons with cognitive impairment. Siewiorek et al [63] from Carnegie Mellon University and the National Science Foundation-funded Quality of Life Technology Center have pioneered the theory and design of virtual coaches $[63,64]$. A virtual coach moves beyond the rote and static reminders of a prompt system. Rather, a virtual coach adequately adapts to the needs of the user. The ideal qualities of a virtual coach as a cognitive aid, as outlined by Siewiorek et al [63], include the virtual coach reducing the number of cues as the user learns, matching the level of support to changes in the user's ability, allowing for caregivers to upload new capabilities to the virtual coach and providing consistent monitoring of adherence to a caregiver's instructions.

\section{Conclusion}

We found that many mHealth app interventions targeting those with cognitive impairment lack health outcomes as a part of their evaluation process and that there is a lack of consensus as to which health outcomes should be used. Of note, the most common health outcomes in this review were cognition, function, quality of life, mood and well-being, and behavioral and psychological symptoms of dementia. These align with outcomes for clinical trials for Alzheimer disease as described in previous systematic reviews [65-67]. From their comprehensive review of the scientific literature in 2017, Bentvelzen et al [67] distilled a list of best practice outcomes for dementia, called the Dementia Outcome Measurement Suite. Our results suggest that a best practice or at least greater consensus is needed around selection of appropriate health outcomes for mHealth app interventions targeting persons with cognitive impairment. The Dementia Outcome Measurement Suite is one best practice guideline that could be considered. The suite has six outcome domains: (1) cognition, (2) staging, (3) function, (4) behavior, (5) delirium, and (6) quality of life [67]. More in-depth discussion of these domains lies outside the scope of this review.

The evidence that use of mHealth app interventions improves the health of people with MCI, Alzheimer disease, and dementia is of limited quality. Evidence met criteria for grade C level of quality as per the OCEBM Levels of Evidence System. Study reports of efficacy were mixed with more than half of the studies (58\%) showing some degree of effectiveness. More RCTs, a larger number of participants, and a design that minimizes bias are needed to better clarify the benefits of these types of interventions.

\section{Acknowledgments}

We would like to thank the support of Indiana University Center for Aging Research, Faculty of the Ruth Lilly Library at Indiana University, the Indiana University Department of Psychiatry and Malaz Boustani, MD, The Richard M Fairbanks Professor of 
Aging Research. This work was supported by the Richard M Fairbanks Professor of Aging Research Chair, Indiana University School of Medicine.

\section{Authors' Contributions}

All authors have read and approved the manuscript. All authors were involved in review and manuscript development.

\section{Conflicts of Interest}

None declared.

\section{Multimedia Appendix 1}

Search Strategy.

[PDF File (Adobe PDF File), 50KB-Multimedia Appendix 1]

\section{References}

1. Ericsson. 2016. Ericsson mobility report URL: https://www.ericsson.com/mobility-report [accessed 2016-11-16] [WebCite Cache ID 6m38yjOGD]

2. Shealy C. Solutions Review: Application Development. 2016 Jul 08. A collection of mobile application development statistics: growth, usage, revenue and adoption URL: https://solutionsreview.com/mobile-application-development/ a-collection-of-mobile-application-development-statistics-growth-usage-revenue-and-adoption/ [accessed 2017-04-02] [WebCite Cache ID 6pPa7wLBU]

3. Levitas D. App Annie. 2016 Feb 10. App forecast: over $\$ 100$ billion in revenue by 2020 URL: https://www.appannie.com/ insights/app-annie-news/app-annie-releases-inaugural-mobile-app-forecast/ [accessed 2017-04-02] [WebCite Cache ID 6pPZSqt6S]

4. HIMSS. 2012 Jan 05. Definitions of mHealth URL: http://www.himss.org/definitions-mhealth [accessed 2016-11-06] [WebCite Cache ID 6louNg2wj]

5. World Health Organization. mHealth: New Horizons for Health Through Mobile Technologies: Second Global Survey on eHealth. Geneva: World Health Organization; 2011.

6. National Institutes of Health. NIH-Wide Strategic Plan Fiscal Years 2016-2020. 2015. URL: https://www.nih.gov/sites/ default/files/about-nih/strategic-plan-fy2016-2020-508.pdf [accessed 2016-11-06] [WebCite Cache ID 6losEYpDB]

7. Hane FT, Robinson M, Lee BY, Bai O, Leonenko Z, Albert MS. Recent progress in Alzheimer's disease research, part 3: diagnosis and treatment. J Alzheimers Dis 2017;57(3):645-665 [FREE Full text] [doi: 10.3233/JAD-160907] [Medline: 28269772]

8. Alzheimer's Association. 2016 Alzheimer's disease facts and figures. Alzheimers Dement 2016 Apr;12(4):459-509. [Medline: 27570871]

9. Oyebode JR, Parveen S. Psychosocial interventions for people with dementia: an overview and commentary on recent developments. Dementia (London) 2016 Jul 04:1. [doi: 10.1177/1471301216656096] [Medline: 27380931]

10. Rebok GW, Ball K, Guey LT, Jones RN, Kim H, King JW, ACTIVE Study Group. Ten-year effects of the advanced cognitive training for independent and vital elderly cognitive training trial on cognition and everyday functioning in older adults. J Am Geriatr Soc 2014 Jan;62(1):16-24 [FREE Full text] [doi: 10.1111/jgs.12607] [Medline: 24417410]

11. Hill NT, Mowszowski L, Naismith SL, Chadwick VL, Valenzuela M, Lampit A. Computerized cognitive training in older adults with mild cognitive impairment or dementia: a systematic review and meta-analysis. Am J Psychiatry 2017 Apr 01;174(4):329-340. [doi: 10.1176/appi.ajp.2016.16030360] [Medline: 27838936]

12. Michael D, Chen S. Serious Games: Games That Educate, Train, and Inform. Mason, OH: Course Technology; 2005.

13. Teipel S, Babiloni C, Hoey J, Kaye J, Kirste T, Burmeister OK. Information and communication technology solutions for outdoor navigation in dementia. Alzheimers Dement 2016 Jun;12(6):695-707 [FREE Full text] [doi:

10.1016/j.jalz.2015.11.003] [Medline: 26776761]

14. Lanza C, Knörzer O, Weber M, Riepe MW. Autonomous spatial orientation in patients with mild to moderate Alzheimer's disease by using mobile assistive devices: a pilot study. J Alzheimers Dis 2014;42(3):879-884. [doi: 10.3233/JAD-140063] [Medline: 24958461]

15. Cotelli M, Manenti R, Zanetti O. Reminiscence therapy in dementia: a review. Maturitas 2012 Jul;72(3):203-205. [doi: 10.1016/j.maturitas.2012.04.008] [Medline: 22607813]

16. Tadaka E, Kanagawa K. Effects of reminiscence group in elderly people with Alzheimer disease and vascular dementia in a community setting. Geriatr Gerontol Int 2007 Jun;7(2):167-173. [doi: 10.1111/j.1447-0594.2007.00381.x]

17. Lai CK, Chi I, Kayser-Jones J. A randomized controlled trial of a specific reminiscence approach to promote the well-being of nursing home residents with dementia. Int Psychogeriatr 2004 Mar;16(1):33-49. [Medline: 15190995]

18. Okumura Y, Tanimukai S, Asada T. Effects of short-term reminiscence therapy on elderly with dementia: A comparison with everyday conversation approaches. Psychogeriatrics 2008 Sep;8(3):133. [doi: 10.1111/j.1479-8301.2008.00236.x] 
19. O'Rourke J, Tobin F, O'Callaghan S, Sowman R, Collins DR. 'YouTube': a useful tool for reminiscence therapy in dementia? Age Ageing 2011 Nov;40(6):742-744. [doi: 10.1093/ageing/afr100] [Medline: 21880626]

20. Hattink BJ, Meiland FJ, Overmars-Marx T, de BM, Ebben PW, van BM, et al. The electronic, personalizable Rosetta system for dementia care: exploring the user-friendliness, usefulness and impact. Disabil Rehabil Assist Technol 2016 Jul;11(1):61-71. [doi: 10.3109/17483107.2014.932022] [Medline: 24989993]

21. Cahill S, Begley E, Faulkner J, Hagen I. "It gives me a sense of independence"? Findings from Ireland on the use and usefulness of assistive technology for people with dementia. Technol Disabil 2007 Jan;19(2):133-142.

22. Kolanowski AM, Litaker M, Buettner L. Efficacy of theory-based activities for behavioral symptoms of dementia. Nurs Res 2005;54(4):219-228. [Medline: 16027564]

23. Tak S, Beck C, Hong S. Feasibility of providing computer activities for nursing home residents with dementia. Nonpharmacol Ther Dement 2013;3(1):1-10 [FREE Full text] [Medline: 25343006]

24. Abraha I, Rimland JM, Trotta FM, Dell'Aquila G, Cruz-Jentoft A, Petrovic M, et al. Systematic review of systematic reviews of non-pharmacological interventions to treat behavioural disturbances in older patients with dementia. The SENATOR-OnTop series. BMJ Open 2017 Mar 16;7(3):e012759 [FREE Full text] [doi: 10.1136/bmjopen-2016-012759] [Medline: 28302633]

25. Yoon DH, Kang D, Kim H, Kim J, Song HS, Song W. Effect of elastic band-based high-speed power training on cognitive function, physical performance and muscle strength in older women with mild cognitive impairment. Geriatr Gerontol Int 2017 May;17(5):765-772. [doi: 10.1111/ggi.12784] [Medline: 27396580]

26. Erickson KI, Voss MW, Prakash RS, Basak C, Szabo A, Chaddock L, et al. Exercise training increases size of hippocampus and improves memory. Proc Natl Acad Sci U S A 2011 Feb 15;108(7):3017-3022 [FREE Full text] [doi: 10.1073/pnas.1015950108] [Medline: 21282661]

27. Bamidis PD, Vivas AB, Styliadis C, Frantzidis C, Klados M, Schlee W, et al. A review of physical and cognitive interventions in aging. Neurosci Biobehav Rev 2014 Jul;44:206-220. [doi: 10.1016/j.neubiorev.2014.03.019] [Medline: 24705268]

28. Moher D, Liberati A, Tetzlaff J, Altman DG. Preferred reporting items for systematic reviews and meta-analyses: the PRISMA statement. PLoS Med 2009 Jul 21;6(7):e1000097 [FREE Full text] [doi: 10.1371/journal.pmed.1000097] [Medline: 19621072]

29. PROSPERO: International Prospective Register of Systematic Reviews. URL: https://www.crd.york.ac.uk/PROSPERO/ about.php?about=about [accessed 2017-08-19] [WebCite Cache ID 6sq33bS5e]

30. Meho LI, Rogers Y. Citation counting, citation ranking, and -index of human-computer interaction researchers: a comparison of Scopus and Web of Science. J Am Soc Inf Sci 2008 Sep;59(11):1711-1726. [doi: 10.1002/asi.20874]

31. Howick J, Chalmers I, Glasziou P, Greenhalgh T, Heneghan C, Liberati A, et al. Centre for Evidence-based Medicine. OCEBM Levels of Evidence URL: http://www.cebm.net/ocebm-levels-of-evidence/ [accessed 2017-08-19] [WebCite Cache ID 6spyPoWpY]

32. Liberati A, Altman DG, Tetzlaff J, Mulrow C, Gøtzsche PC, Ioannidis JP, et al. The PRISMA statement for reporting systematic reviews and meta-analyses of studies that evaluate health care interventions: explanation and elaboration. J Clin Epidemiol 2009 Oct;62(10):e1-e34 [FRE Full text] [doi: 10.1016/j.jclinepi.2009.06.006] [Medline: 19631507]

33. Barnes D, Yaffe K, Belfor N, Jagust WJ, DeCarli C, Reed BR, et al. Computer-based cognitive training for mild cognitive impairment: results from a pilot randomized, controlled trial. Alzheimer Dis Assoc Disord 2009;23(3):205-210. [doi: 10.1097/WAD.0b013e31819c6137] [Medline: 19812460]

34. Chan SC, Chan CC, Derbie AY, Hui I, Tan DG, Pang MY, et al. Chinese calligraphy writing for augmenting attentional control and working memory of older adults at risk of mild cognitive impairment: a randomized controlled trial. J Alzheimers Dis 2017 Jun;58(3):735-746. [doi: 10.3233/JAD-170024] [Medline: 28482639]

35. Tárraga L, Boada M, Modinos G, Espinosa A, Diego S, Morera A, et al. A randomised pilot study to assess the efficacy of an interactive, multimedia tool of cognitive stimulation in Alzheimer's disease. J Neurol Neurosurg Psychiatry 2006 Oct;77(10):1116-1121 [FREE Full text] [doi: 10.1136/jnnp.2005.086074] [Medline: 16820420]

36. Finn M, McDonald S. Computerised cognitive training for older persons with mild cognitive impairment: a pilot study using a randomised controlled trial design. Brain Impair 2011 Dec;12(3):187-199. [doi: 10.1375/brim.12.3.187]

37. Gooding AL, Choi J, Fiszdon JM, Wilkins K, Kirwin PD, van Dyck CH, et al. Comparing three methods of computerised cognitive training for older adults with subclinical cognitive decline. Neuropsychol Rehabil 2016 Oct;26(5-6):810-821. [doi: 10.1080/09602011.2015.1118389] [Medline: 26674122]

38. Han JW, Oh K, Yoo S, Kim E, Ahn K, Son Y, et al. Development of the ubiquitous spaced retrieval-based memory advancement and rehabilitation training program. Psychiatry Investig 2014 Jan;11(1):52-58 [FREE Full text] [doi: 10.4306/pi.2014.11.1.52] [Medline: 24605124]

39. Mansbach WE, Mace RA, Clark KM. The efficacy of a computer-assisted cognitive rehabilitation program for patients with mild cognitive deficits: a pilot study. Exp Aging Res 2017;43(1):94-104. [doi: 10.1080/0361073X.2017.1258256] [Medline: 28067610]

40. Hsiung G, Kupferschmidt A, Naus K, Feldman H, Jacova C. Cognitive and clinical outcomes in a pilot study using a handheld computer game for cognitive training of patients with Mild Cognitive Impairment (MCI). Alzheimers Dement 2009 Jul;5(4):411. [doi: 10.1016/j.jalz.2009.04.1003] 
41. Manera V, Petit P, Derreumaux A, Orvieto I, Romagnoli M, Lyttle G, et al. 'Kitchen and cooking,' a serious game for mild cognitive impairment and Alzheimer's disease: a pilot study. Front Aging Neurosci 2015 Mar;7:24 [FREE Full text] [doi: 10.3389/fnagi.2015.00024] [Medline: 25852542]

42. Merilampi S, Sirkka A, Leino M, Koivisto A, Finn E. Cognitive mobile games for memory impaired older adults. J Assist Technol 2014 Dec 09;8(4):207-223. [doi: 10.1108/JAT-12-2013-0033]

43. Hettinga M, De Boer J, Goldberg E, Moelaert F. Navigation for people with mild dementia. Stud Health Technol Inform 2009;150:428-432. [Medline: 19745347]

44. Yasuda K, Kuwahara N, Kuwabara K, Morimoto K, Tetsutani N. Daily assistance for individuals with dementia via videophone. Am J Alzheimers Dis Other Demen 2013 Aug;28(5):508-516. [doi: 10.1177/1533317513494440] [Medline: 23813611]

45. Imbeault H, Gagnon L, Pigot H, Giroux S, Marcotte N, Cribier-Delande P, et al. Impact of AP@LZ in the daily life of three persons with Alzheimer's disease: long-term use and further exploration of its effectiveness. Neuropsychol Rehabil 2016 Apr 28:1-24. [doi: 10.1080/09602011.2016.1172491] [Medline: 27126266]

46. Meiland FJ, Bouman AI, Sävenstedt S, Bentvelzen S, Davies RJ, Mulvenna MD, et al. Usability of a new electronic assistive device for community-dwelling persons with mild dementia. Aging Ment Health 2012 Jul;16(5):584-591. [doi: 10.1080/13607863.2011.651433] [Medline: 22360649]

47. Leng FY, Yeo D, George S, Barr C. Comparison of iPad applications with traditional activities using person-centred care approach: impact on well-being for persons with dementia. Dementia (London) 2014 Mar 01;13(2):265-273. [doi: 10.1177/1471301213494514] [Medline: 24339097]

48. Tyack C, Camic PM, Heron MJ, Hulbert S. Viewing art on a tablet computer. J Appl Gerontol 2015 Dec 01:733464815617287. [doi: 10.1177/0733464815617287] [Medline: 26675353]

49. Astell AJ, Joddrell P, Groenewoud H, de Lange J, Goumans M, Cordia A, et al. Does familiarity affect the enjoyment of touchscreen games for people with dementia? Int J Med Inform 2016 Jul;91:e1-e8. [doi: 10.1016/j.ijmedinf.2016.02.001] [Medline: 26897552]

50. Hsu C, Lee E, Vahia I. Tablet computer use in reducing neuropsychiatric symptoms in patients with dementia. J Am Geriatr Soc 2016 May;64(S1):S90.

51. Vahia IV, Kamat R, Vang C, Posada C, Ross L, Oreck S, et al. Use of tablet devices in the management of agitation among inpatients with dementia: an open-label study. Am J Geriatr Psychiatry 2016 Aug 04:860-864. [doi:

10.1016/j.jagp.2016.07.011] [Medline: 27746070]

52. Van der Ploeg ES, Eppingstall B, O'Connor DW. Internet video chat (Skype) family conversations as a treatment of agitation in nursing home residents with dementia. Int Psychogeriatr 2015 Nov 12;28(04):697-698. [doi: 10.1017/s1041610215001854]

53. Lim FS, Wallace T, Luszcz MA, Reynolds KJ. Usability of tablet computers by people with early-stage dementia. Gerontology 2013;59(2):174-182. [doi: 10.1159/000343986] [Medline: 23257664]

54. Vidoni ED, Watts AS, Burns JM, Greer CS, Graves RS, Van Sciver A, et al. Feasibility of a memory clinic-based physical activity prescription program. J Alzheimers Dis 2016 Apr 21;53(1):161-170. [doi: 10.3233/JAD-160158] [Medline: 27104905]

55. Federal Trade Commission. 2015 Jan 06. Lumosity to pay $\$ 2$ million to settle FTC deceptive advertising charges for its "brain training" program URL: https://www.ftc.gov/news-events/press-releases/2016/01/ lumosity-pay-2-million-settle-ftc-deceptive-advertising-charges [accessed 2017-04-02] [WebCite Cache ID 6pPYtU3fr]

56. Cortez NG, Cohen IG, Kesselheim AS. FDA regulation of mobile health technologies. N Engl J Med 2014 Jul 24;371(4):372-379. [doi: 10.1056/NEJMhle1403384] [Medline: 25054722]

57. US Department of Health and Human Services Food and Drug Administration, Center for Devices and Radiological Health, Center for Biologics Evaluation and Research. Mobile Medical Applications: Guidance for Industry and Food and Drug Administration Staff. Silver Spring, MD: Food and Drug Administration; Feb 09, 2015.

58. Blenner SR, Köllmer M, Rouse AJ, Daneshvar N, Williams C, Andrews LB. Privacy policies of android diabetes apps and sharing of health information. JAMA 2016 Mar 08;315(10):1051-1052. [doi: 10.1001/jama.2015.19426] [Medline: 26954415]

59. Andrews L. I Know Who You Are and I Saw What You Didocial Networks and the Death of Privacy. New York: Free Press; 2012.

60. Fisk A, editor. Designing for Older Adults: Principles and Creative Human Factors Approaches. 2nd ed. Boca Raton, FL: CRC Press; 2009.

61. Holden RJ, Carayon P, Gurses AP, Hoonakker P, Hundt AS, Ozok AA, et al. SEIPS 2.0: a human factors framework for studying and improving the work of healthcare professionals and patients. Ergonomics 2013;56(11):1669-1686 [FREE Full text] [doi: 10.1080/00140139.2013.838643] [Medline: 24088063]

62. Shiffman S, Stone AA, Hufford MR. Ecological momentary assessment. Annu Rev Clin Psychol 2008;4:1-32. [Medline: 18509902]

63. Siewiorek D, Smailagic A, Dey A. Architecture and applications of virtual coaches. Proc IEEE 2012 Aug;100(8):2472-2488. [doi: 10.1109/JPROC.2012.2200560]

64. Carnegie Mellon University. Quality of Life Technology Center URL: http://www.cmu.edu/qolt/ [accessed 2017-04-02] [WebCite Cache ID 6pPYjHPQ2] 
65. Schneider LS, Mangialasche F, Andreasen N, Feldman H, Giacobini E, Jones R, et al. Clinical trials and late-stage drug development for Alzheimer's disease: an appraisal from 1984 to 2014. J Intern Med 2014 Mar;275(3):251-283 [FREE Full text] [doi: 10.1111/joim.12191] [Medline: 24605808]

66. Cooper C, Mukadam N, Katona C, Lyketsos CG, Blazer D, Ames D, et al. Systematic review of the effectiveness of pharmacologic interventions to improve quality of life and well-being in people with dementia. Am J Geriatr Psychiatry 2013 Feb;21(2):173-183. [doi: 10.1016/j.jagp.2012.10.018] [Medline: 23343491]

67. Bentvelzen A, Aerts L, Seeher K, Wesson J, Brodaty H. A comprehensive review of the quality and feasibility of dementia assessment measures: the dementia outcomes measurement suite. J Am Med Dir Assoc 2017 Mar 07:1. [doi: 10.1016/j.jamda.2017.01.006] [Medline: 28283381]

\author{
Abbreviations \\ ADL: activities of daily living \\ FDA: Food and Drug Administration \\ GBS: Gottfries-Brane-Steen \\ MCI: mild cognitive impairment \\ MMSE: Mini-Mental Status Examination \\ NIH: National Institutes of Health \\ OCEBM: Oxford Centre for Evidence-based Medicine \\ PRISMA: Preferred Reporting Items for Systematic Review and Meta-Analyses \\ RCT: randomized controlled trials
}

\author{
Edited by G Eysenbach; submitted 01.04.17; peer-reviewed by P Bamidis, B Davis, SFA Shah, M Ashford; comments to author \\ 24.04.17; revised version received 15.07.17; accepted 01.08.17; published 30.08.17 \\ Please cite as: \\ Bateman DR, Srinivas B, Emmett TW, Schleyer TK, Holden RJ, Hendrie HC, Callahan CM \\ Categorizing Health Outcomes and Efficacy of mHealth Apps for Persons With Cognitive Impairment: A Systematic Review \\ J Med Internet Res 2017;19(8):e301 \\ URL: http://www.jmir.org/2017/8/e301/ \\ doi: 10.2196/jmir.7814 \\ PMID: 28855146
}

CDaniel R Bateman, Bhavana Srinivas, Thomas W Emmett, Titus K Schleyer, Richard J Holden, Hugh C Hendrie, Christopher M Callahan. Originally published in the Journal of Medical Internet Research (http://www.jmir.org), 30.08.2017. This is an open-access article distributed under the terms of the Creative Commons Attribution License (https://creativecommons.org/licenses/by/4.0/), which permits unrestricted use, distribution, and reproduction in any medium, provided the original work, first published in the Journal of Medical Internet Research, is properly cited. The complete bibliographic information, a link to the original publication on http://www.jmir.org/, as well as this copyright and license information must be included. 